\title{
КОЛЕБАТЕЛЬНАЯ РЕЛАКСАЦИЯ И ГОРЯЧАЯ ЛЮМИНЕСЦЕНЦИЯ СИСТЕМ С КВАДРАТИЧНЫМ ВИБРОННЫМ ВЗАИМОДЕЙСТВИЕМ
}

\author{
V. HIZNJAKOV. VONKERELAKSATSIOON JA KUUM LUMINESTSENTS VIBROONSE INTER- \\ AKTSIOONI RUUTSOLTUVUSE KORRAL \\ V. HIZHNYAKOV. VIBRATIONAL RELAXATION AND HOT LUMINESCENCE IN, SYSTEMS WITH \\ QUADRATIC VIBRONIC COUPLING
}

В кристаллах и молекулярных системах электронные и колебательные степени свободы существенно связаны. Поэтому первичные электронные переходы, возникающие при различных физических процессах в них, сопровождаются возбуждением колебаний. Последующая колебательная релаксация влияет, в свою очередь, на вторичные электронные переходы. В ряде случаев это влияние имеет определяющее для процесса значение. В качестве конкретных примеров электронных переходов в неравновесном (горячем) колебательном состоянии можно привести горячую люминесценцию $\left[{ }^{1,2}\right]$ и горячую передачу электронного возбуждения $\left[{ }^{3}\right]$. Можно думать, что такие переходы существенны в некоторых химических превращениях.

Из сказанного ясно, что разработка теории колебательной релаксации, сопровождающей электронные переходы, и изучение ее проявлений представляют собой актуальную задачу. Особенность этой задачи в том, что в ней существенны колебательные характеристики центра в двух разных электронных состояниях - в конечном (возбужденном), в котором происходит колебательная релаксация, и в исходном (основном), от которого зависит начальное условие релаксации. Сказанное следует уже из общего определения колебательной матрицы плотности релаксирующей системы $(t)=\exp \left(-i t H_{1}\right) \varrho_{0} \exp \left(i t H_{1}\right)$, где $\varrho_{0}-$ колебательная матрица плотности в начальный момент времени, которая при белом возбуждении равна $\varrho_{0}=\exp \left(-H_{0} / k T\right) \times\left[\operatorname{Sp} \exp \left(-H_{0} / k T\right)\right]^{-1}$, $H_{0}$ и $H_{1}$ - колебательные гамильтонианы основного и возбужденного электронных состояний, $\hbar=1$. В пределе $t \rightarrow \infty \quad \varrho(t) \rightarrow \varrho_{1}=$ $=\exp \left(-H_{1} / k T\right)\left[\mathrm{Sp} \exp \left(-H_{1} / k T\right)\right]^{-1}$.

Простейшими характеристиками релаксирующей электронно-колебательной системы, определяемыми матрицей плотности $\varrho(t)$, являются величины

$$
\begin{gathered}
\bar{q}_{t}=\mathrm{Sp}\left[q_{\varrho}(t)\right]=\left\langle q(t)_{1}\right\rangle_{0}, \\
\overline{\delta q_{t}^{2}}=\operatorname{Sp}\left[\left(q-\bar{q}_{t}\right)^{2} \varrho(t)\right]=\left\langle\hat{q}_{t}^{2}\right\rangle_{0} .
\end{gathered}
$$

Здесь $q-$ некоторый оператор системы, $\hat{q}_{t}=q(t)_{1}-\bar{q}_{t}, \quad q(t)_{1}=$ $=\exp \left(i t H_{1}\right) q \exp \left(-i t H_{1}\right),\langle\ldots\rangle_{k}=\mathrm{Sp}\left(\ldots \mathrm{Q}_{k}\right), k=0,1$. Если под $q$ понимать конфигурационную координату центра, то $\bar{q}_{k}$ определяет релаксацию ее среднего значения, а $\left\langle\hat{q}_{t}{ }^{2}\right\rangle_{0}-$ временное поведение ее квадра- 
тичной флуктуации. Аналогичным образом можно определить и временную зависимость флуктуаций более высокого порядка, а также произведений различных операторов.

Если возмущение, вызывающее электронный переход, не белое, то существенно знать и величины типа $\left\langle q(t){ }_{1} q^{\prime}\right\rangle_{0}$. В частности, при монохроматическом возбуждении огибающая суммарного спектра горячей и обынной люминесценции равна [ $\left.{ }^{4}\right]$

$$
I\left(\Omega, \Omega_{0}\right) \sim \int_{0}^{\infty} d t\left(2 \pi \sigma_{t}^{2}\right)^{-1 / 2} \exp \left[-\gamma t-\left(\Omega-\Omega_{t}\right)^{2} / 2 \sigma_{t}^{2}\right],
$$

где $\Omega_{0}$ и $\Omega$ - частоты возбуждения и излучения, $\gamma$ - константа радиационного распада,

$$
\begin{gathered}
\Omega_{t}=\bar{V}_{t}+\left(\Omega_{0}-\bar{V}_{0}\right)\left\langle\left\{\hat{V}_{t}, . \hat{V}_{0}\right\}\right\rangle_{0} / 2\left\langle\hat{V}_{0}^{2}\right\rangle_{0}, \\
\sigma_{t}^{2}=\left\langle\hat{V}_{t}^{2}\right\rangle_{0}-\left\langle\left\{\hat{V}_{t}, \hat{V}_{0}\right\}\right\rangle_{0}^{2} / 4\left\langle\hat{V}_{0}^{2}\right\rangle_{0},
\end{gathered}
$$

фигурные скобки означают антикоммутатор, $V=H_{1}-H_{0}$. Отметим, что величины типа фигурирующих в (4) и (5) определяют и огибающую зависящего от времени (переходного) спектра резонансного вторичного свечения [ $\left.{ }^{5}\right]$.

Приведенные выше величины описывают колебательную релаксацию, если система имеет неограниченное число колебательных степеней свободы и обладает квазинепрерывным спектром колебательных возбуждений (предполагается, что гамильтонианы $H_{0}$ и $H_{1}$ эрмитовы и не зависят от времени). В противном случае, ка̨к известно, система не релаксирует, а совершает циклы Пуанкаре. В случае примесного центра нужным свойством обладает уже гармоническая решетка с квазинепрерывным фононным спектром. Эта простая модельная система, допускающая последовательное динамическое рассмотрение релаксации, и будет использоваться в данной работе.

Приведенные выше релаксационные характеристики (3)-(5) в случае гармонической решетки и линейного вибронного взаимодействия легко вычисляются [ $\left.{ }^{5}\right]$. Такое приближение достаточно хорошо оправдано, если вибронное взаимодействие слабое. Если же это не так, то необходим учет и членов, квадратичных по смещениям. При этом задача расчета релаксационных характеристик существенно усложняется из-за перепутывания нормальных колебаний при электронном переходе (называемого также вращением Душинского). В данной работе мы покажем, что эффект перепутывания нормальных координат в рассматриваемой задаче может быть точно учтен, если основное и возбужденное электронные состояния не вырождены.

Без ограничения общности квадратичное по смещениям ядер $Q$ вибронное взаимодействие можно представить в виде

$$
V=V_{L}+A_{1}+Q+\frac{1}{2} Q+B Q
$$

Если электронные состояния не вырождены, то $A_{1}$ и $Q-$ одностолбцовые матрицы с $n$ элементами, $A_{1}{ }^{+}$и $Q^{+}-$соответствующие сопряженные матрицы, $B$ - эрмитова матрица $n \times n, n-$ число учитываемых конфигурационных координат. Разложение матрицы конфигурационных координат $Q$ по нормальным координатам $x_{i}$ и $y_{j}$ основного и возбужденного электронных состояний имеет вид

$$
Q=\bar{Q}_{0}+\sum_{i} S_{0 i} x_{i}=\sum_{j} S_{1 j} y_{j}
$$


причем

$$
y_{j}=y_{0 j}+\sum_{i} x_{i} c_{i j}
$$

$S_{0 i}$ и $S_{1 j}$ - одностолбцовые матрицы с $n$ элементами, связанные соотношением $S_{0 i}=\sum_{j} S_{1 j} c_{i j}$, где

$$
\begin{aligned}
& c_{i j}=S_{1 j}^{+} B S_{0 i}\left(\omega_{j}^{2}-\omega_{i}^{2}\right)^{-1}, \\
& \sum_{i} S_{0 i} S_{0 i}^{+}\left(\omega_{j}^{2}-\omega_{i}^{2}\right)^{-1}=\sum_{j} S_{1 j} S_{1 j}^{+}\left(\omega_{j}^{2}-\omega_{i}^{2}\right)^{-1}=B^{-1},
\end{aligned}
$$

$\omega_{i}$ и $\omega_{j}-$ частоты колебаний, соответствующие нормальным координатам $x_{i}$ и $y_{j}$. Подставим формулы (5)-(9) в (4) и (5). После простых вычислений получим

$$
\begin{gathered}
\Omega_{t}=\bar{V}_{t}+\left(\Omega_{0}-\bar{V}_{0}\right)\left\langle\left\{A_{t}^{+} \hat{Q}_{t}, \hat{Q}_{0}^{+} A_{0}\right\}\right\rangle_{0} / 2 A_{0}^{+}\left\langle\hat{Q}_{0} \hat{Q}_{0}^{+}\right\rangle_{0} A_{0} \\
\sigma_{t}^{2}=A_{t}^{+}\left\langle\hat{Q}_{t} \hat{Q}_{t}^{+}\right\rangle_{0} A_{t}-\left\langle\left\{A_{t}^{+} \hat{Q}_{t}, \hat{Q}_{0}^{+} A_{0}\right\}\right\rangle_{0}^{2} / 4 A_{0}^{+}\left\langle\hat{Q}_{0} \hat{Q}_{0}^{+}\right\rangle_{0} A_{0}
\end{gathered}
$$

где

$$
\begin{aligned}
& \bar{V}_{t}=V_{L}+A_{1}^{+} \bar{Q}_{t}+\frac{1}{2} \bar{Q}_{t}^{+B} \bar{Q}_{t}, \\
& \bar{Q}_{t}=\sum_{j} S_{1 j} S_{1 j}^{+} A_{0} \omega_{j}^{-2} \cos \omega_{j} t
\end{aligned}
$$

- классические зависимости $V$ и $Q$ от времени, $A_{t}=A_{1}+B \bar{Q}_{t}$,

$$
\begin{gathered}
\left\langle\hat{Q}_{t} \hat{Q}_{t}^{+}\right\rangle_{0}=\sum_{i} \omega_{i}^{-1}\left(\bar{n}_{i}+1 / 2\right) D_{i}^{+}(t) S_{0 i} S_{0 i}^{+} D_{i}(t), \\
\left\langle\left\{A_{t}^{+} \hat{Q}_{t}, \hat{Q}_{0}^{+} A_{0}\right\}\right\rangle_{0}=\sum_{i} \omega_{i}^{-1}\left(2 \bar{n}_{i}+1\right) A_{t}^{+} S_{0 i} S_{0 i}^{+} \operatorname{Re} D_{i}(t) A_{0},
\end{gathered}
$$

$\bar{n}_{i}=\left[\exp \left(-\omega_{i} / k T\right)-1\right]^{-1}$. Здесь веедена матричная функция

$$
D_{i}(t)=B \sum_{j} S_{1 j} S_{1 j}^{+}\left(\omega_{j}^{2}-\omega_{i}^{2}\right)^{-1}\left(\cos \omega_{j} t+i\left(\omega_{i} / \omega_{j}\right) \sin \omega_{j} t\right) .
$$

Эту функцию можно вычислить, если перейти от суммирования по $j$ к интегрированию по частоте. При этом вклад полюса подынтегрального выражения следует определить из второго уравнения в (10). Переходя также от суммирования по $i$ к интегрированию по частоте, получим

$$
\begin{gathered}
\bar{Q}_{t}=\frac{1}{2 \pi} \int_{-\infty}^{\infty} d \omega e^{i \omega t} \omega^{-1} \operatorname{Im} \oiint_{1}(\omega) A_{0}, \\
\left\langle\hat{Q}_{t} \hat{Q}_{t}^{+}\right\rangle_{0}=\frac{1}{\pi} \int_{0}^{\infty} d \omega(2 n(\omega)+1) D+(\omega, t)\left[\operatorname{Im} \varpi_{0}(\omega)\right] D(\omega, t), \\
\left\langle\left\{A_{t}^{+} \hat{Q}_{t}, \hat{Q}_{0}^{+} A_{0}\right\}\right\rangle_{0}=\frac{2}{\pi} \int_{0}^{\infty} d \omega(2 n(\omega)+1) A_{t}^{+}\left[\operatorname{Im} \varpi_{0}(\omega)\right] \operatorname{Re} D(\omega, t) A_{0},
\end{gathered}
$$

где $n(\omega)=[\exp (-\omega / k T)-1]^{-1}$, 


$$
D(\omega, t)=e^{i \omega t}\left(I+B \oiint_{1}(\omega)\right)-R(\omega, t),
$$

$$
\begin{gathered}
R(\omega, t)=i B e^{i \omega t} \operatorname{Im} \circlearrowleft_{1}(\omega)+\frac{1}{\pi} B \int_{-\infty}^{\infty} d \omega^{\prime}\left(\omega^{2}-\omega^{\prime 2}\right)^{-1}\left(\left|\omega^{\prime}\right| \cos \omega^{\prime} t+\right. \\
\left.+i \omega \sin \omega^{\prime} t\right) \operatorname{Im} \varpi_{1}\left(\omega^{\prime}\right) .
\end{gathered}
$$

Здесь введены матрицы динамических функций Грина в основном и возбужденном электронных состояниях, равные

$$
\begin{aligned}
& \circlearrowleft_{0}(\omega)=\sum_{i} S_{0 i} S_{0 i}^{+}\left(\omega^{2}-\omega_{i}^{2}-i \omega \varepsilon\right)^{-1}, \\
& \circlearrowleft_{1}(\omega)=\sum_{j} S_{1 j} S_{1 j}^{+}\left(\omega^{2}-\omega_{j}^{2}-i \omega \varepsilon\right)^{-1}
\end{aligned}
$$

( $\varepsilon$ - бесконечно малая положительная величина); они связаны между собой уравнением Дайсона

$$
\mathscr{G}_{1}(\omega)=\mathscr{G}_{0}(\omega)\left(I+B \mathscr{G}_{1}(\omega)\right),
$$

где $I$ - единичная матрица $n \times n$.

Используя интегральное представление резольвенты

$$
\left(\omega^{2}-\omega^{\prime 2}\right)^{-1}=\omega^{-1} \int_{0}^{\infty} \sin (\omega \tau) \cos \left(\omega^{\prime} \tau\right) \exp (-\varepsilon \tau) d \tau,
$$

$R(\omega, t)$ можно преобразовать к виду

$$
R(\omega, t)=B \int_{0}^{\infty} d \tau \sin (\omega \tau)\left[g_{1}(t+\tau)+\omega^{-1} g_{2}(t+\tau)\right] \exp (-\varepsilon \tau),
$$

где

$$
\begin{aligned}
& g_{1}(t)=\frac{1}{2 \pi} \int_{-\infty}^{\infty} e^{i \omega t} \operatorname{Im} \mathscr{G}_{1}(\omega) d \omega, \\
& g_{2}(t)=\frac{1}{2 \pi} \int_{-\infty}^{\infty} e^{i \omega t} \omega \operatorname{Im} \mathscr{G}_{1}(\omega) d \omega .
\end{aligned}
$$

Подставляя эти формулы в (18) и учитывая (22), получаем

$$
\begin{aligned}
\left\langle\hat{Q}_{t} \hat{Q}_{t}^{+}\right\rangle_{0} & =\left\langle Q Q^{+}\right\rangle_{1}+\frac{1}{\pi} \int_{0}^{\infty} d \omega(2 n(\omega)+1)\left\{R^{+}(\omega, t)\left[\operatorname{Im} \circlearrowleft_{0}(\omega)\right] R(\omega, t)-\right. \\
& \left.-2 \operatorname{Re} e^{i \omega t} R^{+}(\omega, t)\left[\operatorname{Im} \varpi_{0}(\omega)\right]\left(I+B \mathscr{G}_{1}(\omega)\right)\right\} .
\end{aligned}
$$

Заметим, что в случае квазинепрерывного фононного спектра, как это следует из формул $(23)-(25), g_{1}(t), g_{2}(t), R(\omega, t) \rightarrow 0$ при $t \rightarrow \infty$. Поэтому в соответствии с отмеченным выше соотношением $Q(\infty)=Q_{1}$ имеем $\left\langle\hat{Q}_{\infty} \hat{Q}_{\infty}^{+}\right\rangle_{0}=\operatorname{Sp}\left(\hat{Q}_{0} \hat{Q}_{0}^{+} \varrho_{\infty}\right)=\left\langle Q Q^{+}\right\rangle_{1}$.

Формулы (11), (12), (17), (19)-(26) решают рассматриваемую задачу. Действительно, зная (ூ) $(\omega)$ (нахождение этой функции есть задача локальной динамики решетки, решаемая методом Лифшица), по формуле (22) можно найти $\mathfrak{G}_{1}(\omega)$, а затем по формулам (11), (12), $(17),(19),(20),(23)-(26)-$ и все искомые величины. Следует, однако, отметить, что использование полученных формул для конкретных рас- 
четов возможно, если при электронном переходе в примесном центре силовые постоянные изменяются лишь для небольшого числа $\sim n$ сфер вокруг центра. Это предположение обычно выполняется.

Автор признателен Г. Завту за обсуждение.

\section{ЛИТЕРА Т У А}

1. Rebane, K., Hizhnyạov, V., Tehver, I., ENSV TA Toimet., Füüs. Matem., 16, № 2, 207-232 (1967).

2. S a a r i, P., R e ba n e, K., Solid State Communs, 7, № 12, 887-890 (1969); $\mathrm{R}$ e b a n e, K., S a a r i, P., J. Luminescence, 16, № 3, 223-243 (1978).

3. Техвер И. Ю., Хи жняков В. В., Ж. эксперим. и теор. физ., 69, вып. 2(8), $599-610(1975)$

4. H izh ny a kov, V., K ink, R., Selg, M., Sherman, A., In: Proceedings of the Second International Symposium «Ultrafast Phenomena in Spectroscopy», Reinhardsbrunn G.D.R., 2, 1980, p. 468-472.

5. Хи жняков В., Реб ан е И., Изв. АН ЭССР, Физ. Матем., 26, № 3, 260-280 (1977).

Институт физики

Академии наук Эстонской ССР
Поступила в редакцию $26 / \mathrm{X} 1981$

EESTI NSV TEADUSTE AKADEEMIA TOIMETISED. 31. KOIDE FOOSIKA * MATEMAATIKA. 1982, NR. 1

ИЗВЕСТИЯ АКАДЕМИИ НАУК ЭСТОНСКОИ ССР. ТОМ З1 ФИЗИКА * МАТЕМАТИКА. 1982, №, 1

A. ШЕРМАH

удк 535.375

\section{ОБ АВТОЛОКАЛИЗАЦИИ ЭКСИТОНОВ И ДЫРОК В КРИСТАЛЛЕ КСЕНОНА}

A. SERMAN. EKSITONIDE JA AUKUDE AUTOLOKALISATSIOON KSENOONIKRISTALLIS

A. SERMAN. ON THE SELF-TRAPPING OF EXCITONS AND HOLES IN XENON CRYSTAL

(Представил В. Хижняков)

Как известно, в кристалле Хе имеет место явление автолокализации экситонов (в качестве обзора см., напр., $\left[{ }^{1}\right]$ ). Автолокализации же дырок в этих кристаллах до последнего времени обнаружить не удавалось $\left[{ }^{2}\right]$. Этим фактам можно дать простое объяснение, если предположить, что константы деформационного потенциала электрона $\sigma_{e}$ и дырки $\sigma_{h}$ имеют одинаковый знак. В этом случае перекрытие областей деформации кристалла вокруг электрона и дырки приводит к усилению взаимодействия с фононами каждой из квазичастиц, т. е. связывание их в экситон создает более благоприятные условия для автолокализации. При значительном перекрытии областей деформации, $\sigma_{e}=\sigma_{h}$ и равенстве эффективных масс электрона и дырки, $m_{e}=m_{h}$, константа деформационного потенциала экситона примерно вдвое превышает $\sigma_{h}$. Полагая $\Lambda \ll R$ ( $\Lambda$ - высота барьера автолокализации для экситона, отсчитываемая от дна $1 s$-зоны, $R$ - постоянная Ридберга экситона), можно 